\title{
Wortbildung mit exogenen Einheiten
}

\begin{abstract}
Productive word formation of present-day German lexemes with exogenous elements, particularly confixes, has increased notably over the last few years. Consequentially these formations containing foreign elements have also attracted a great deal of attention among word formation researchers. Working towards an encyclopedic compilation of exogenous elements in German word formation it is demanded to crucially analyze these foreign elements in terms of etymological, structural, semanticofunctional and textlinguistico-pragmatic criteria. This article provides insight into these aspects of analysis by examples of word-formation using exogenous elements such as -itis, -manie, -phob, and -aholic.
\end{abstract}

In der deutschen Gegenwartssprache, insbesondere in Pressetexten, findet man mehr und mehr Wortbildungen, die entweder völlig aus exogenen Einheiten bestehen, wie suboptimal ('weniger gut'), Populistenduo (Benennung für das neue Linksbündnis von PDS und WASG), Minijob, Islamophobie, Super-Uni, oder die Hybridbildungen mit indigenen Einheiten darstellen, wie Umfrageritis, Koma-Saufen, Multi-Kulti-Träume, Googleisierung, Fußballologe.

In der Wortbildungsforschung haben solche Fremdwortbildungen auf Grund ihrer Produktivität im Sprachgebrauch in den letzten Jahren mehr und mehr Aufmerksamkeit erfahren, und exogene Wortbildungseinheiten, die bisher nur am Rande betrachtet wurden, rücken nun in den Mittelpunkt des Forschungsinteresses. In den vergangenen Jahren sind eine ganze Reihe von Monographien und Aufsätzen zu einzelnen Fremdwortbildungsmorphemen erschienen (z. B. Nortmeyer 1987, 2000; Schmidt 1987; Kirkness 1996; Klosa 1996; Ruf 1996; Schmitt 1996; Hoppe 1998, 1999, 2000; Kinne 2000; Feine 2003), und auch in den neueren Wortbildungslehren werden sie in die Betrachtung der Wortbildungseinheiten und -arten einbezogen ( $\mathrm{z}$. B. Fleischer/Barz 1995; Simmler 1998; Altmann/Kemmerling 2000; Erben 2000; Donalies 2002). Dennoch besteht weiterer, dringender Bedarf, solche Fremdwortbildungseinheiten zu analysieren und zu beschreiben hinsichtlich 
ihres Status, ihrer Kombinationsmöglichkeiten, ihres Funktionspotentials und ihres Auftretens in bestimmten Kommunikationsbereichen.

Ihre Erforschung ist zugleich in einem erweiterten Rahmen zu sehen, im Rahmen der Internationalismen- bzw. Europäismenforschung, denn viele dieser Fremdeinheiten sind auch in anderen Sprachen produktiv und können als Inter- bzw. Euromorpheme (vgl. Müller 2000) bezeichnet werden. Für das Französische liegt mit dem „Dictionnaire des structures du vocabulaire savant" von Cottez bereits eine Dokumentation solcher gebundener Fremdwortbildungselemente vor, für das Deutsche muss sie noch geschaffen werden. Die Erstellung eines Lexikons der deutschen Lehnwortbildung war schon in den 80er Jahren des vergangenen Jahrhunderts Ziel einer Arbeitsgruppe am Institut für deutsche Sprache in Mannheim, konnte damals aber nicht realisiert werden (vgl. Hoppe et al. (Hg.) 1987).

Für eine lexikographische Darstellung von gebundenen Fremdwortbildungselementen ist im Vorfeld noch ein immenser Aufwand an Analysen zu leisten.

Analysiert man Fremdwortbildungen, um die sie konstituierenden Wortbildungseinheiten zu erfassen, stößt man neben den Einheiten Grundmorphem und Wortbildungsaffix (Präfix, Suffix und Zirkumfix) auf eine Einheit, die als Konfix bezeichnet wird: Schokaholic, Gastro-Bus, ossiphob, Krupto-Single.

Der Terminus Konfix wurde 1985 von Fischer als "gebundenes Grundmorphem" in die Diskussion gebracht, nachdem bereits Martinet (1980) die Kombination von gebundenen Morphemen als confixation bezeichnet und Kocourek (1982:94) Konfixe als "(confixes), c' est-à-dire racines savantes antérieures (ex. logo-) on postérieures (ex. -logue)" bestimmt hatte.

Seitdem sind verschiedene Definitionsansätze publiziert worden, nach denen Konfixe produktive basis- und/oder kompositionsgliedfähige Einheiten sind. Von den Wörtern unterscheiden sie sich durch ihr lediglich gebundenes Auftreten, von den Affixen durch ihre Basisfähigkeit und z. T. durch Positionsvariabilität. Einige Konfixe können sowohl als Erst- als auch als Zweitglied von Wortbildungsprodukten auftreten (thermophil - Philanthrop, phobisch - ossiphob). In der Regel sind Konfixe Fremdmorpheme. Fleischer (1995) bezeichnet zwar auch indigene Morpheme, wie z. B. stief- und -wart als solche. Sie sind hinsichtlich ihres sehr geringen Produktivitätsgrades aber nicht mit den exogenen Konfixen vergleichbar.

Bezüglich der morphologischen Seite von Konfixen gibt es keinen Dissens, nicht ganz so übereinstimmend ist die Position zur semantischen Seite. Die einen sprechen den Konfixen im Gegensatz zu den Affixen eine lexikalisch-begriffliche Bedeutung zu, die anderen sind der Auffassung, dass auch Affixe eine ausgeprägte Eigensemantik besitzen können und dass nach dem 
semantischen Kriterium keine eindeutige Abgrenzung möglich ist (Müller 2005: 29; 2000: 123).

Im Inventar der Wortbildungseinheiten werden Konfixe unterschiedlich positioniert. Sie nehmen entweder „eine Art Zwischenstellung zwischen freien Morphemen und den Affixen ein" (Grimm 1997: 277) oder werden nach Schmidt (1987: 50) neben den Affixen unter dem Oberbegriff Kombinem geordnet oder sind nach Müller (2000: 125) initial und/oder terminal auftretende gebundene Affixe neben den initial positionierten Präfixen und den terminal positionierten Suffixen.

Abgesehen von der Statusfrage solcher Fremdeinheiten sind Wortbildungsprodukte mit fremden Elementen unter einer ganzen Reihe anderer Aspekte zu untersuchen, so z. B. unter ausdrucksseitig-morphologischem, semantisch-funktionalem und textlinguistisch-pragmatischem Aspekt.

Im Folgenden sollen diese Aspekte am Beispiel einiger Fremdelemente kurz angerissen und gezeigt werden, welch breites Spektrum an Wortbildungen möglich ist und in welcher Hinsicht solche Wortbildungen detailliert erforscht werden müssten.

Ausdrucksseitig-morphologische Analysen zeigen die vielfältigen Kombinationsmöglichkeiten von Fremdelementen im Rahmen der bekannten Wortbildungsmuster sowie eine Vielzahl von Struktur- und Graphievarianten. Die Fremdelemente treten als Konstituenten in Komposita und Derivaten in den schon genannten Positionen initial und / oder terminal auf: karnevalphob, Phobie, Dromomanie, Autodrom, austrophil, Neoaustro, Spielaholic. Auch Kontaminationen treten auf, wie Walkmanie (Walkman-Manie), austrojanisches (austro-trojanisches) Pferd, und Kombinationen von Kurzwort und Fremdelement, wie PON-oholic (PON = Polski Owczarek Nizinny), Pc-oholik. Obwohl die Konstituente -oholic/-aholic immer Personen bezeichnet, treten - oholic-Bildungen auf, die - wahrscheinlich zur Verdeutlichung einer Personenbezeichnung - mit -er/-erin deriviert sind: Schokoholiker, Sexaholiker, Fleischoholikerin.

Eine morphologische Vielfalt ergibt sich weiterhin durch Struktur- und Graphievarianten:

suffixlos vs. suffixhaltig:

euorophob-europhobisch;

indogenes Suffix vs. exogenes Suffix:

Bourgeoisophobe - Bourgeoisophobus, kryptisch - kryptoid;

Pluralform vs. Singularform der ersten Konstituente:

Ämterphobie - Amtsphobie;

Nullfuge vs. Fugenelement:

Frackphobie - Frackophobie, Sex-Manie - Sexomanie, Millennium-Manie - Millenniumsmanie, Kryptästhesie - Kryptovulkanismus; 
Vollformkonstituente vs. Kurzformkonstituente:

Diana-Manie - Di-Manie, Dinosaurier-Manie - Dinomanie, Sauriermanie - Sauromanie;

Zusammenschreibung der Konstituenten vs. Getrenntschreibung vs. Bindestrichschreibung vs. Binnengroßschreibung:

Austropop - Austro_Pop - Austro_Pop - Austro_lassic, Callboy - Call_boy - Call-boy-Cally a-Nummer.

In semantisch-funktionaler Hinsicht ist festzustellen, dass bei den Fremdelementen im Vergleich zu ihrer Bedeutung in der Herkunftssprache in der Regel ein Bedeutungswandel stattgefunden hat bzw. immer noch stattfindet. Eine Reihe von Fremdelementen, die zunächst nur in Termini der medizinischen Fachsprache zu finden waren, werden nun verstärkt zur Bildung neuer Wörter in der Gemeinsprache verwendet, wie z. B. -itis, -manie und -phobie, wobei -manie und -phobie bereits Derivate sind. Als medizinische Termini zählen $z$. B.

Meningitis (Hirnhautentzündung), Arthritis (Gelenkentzündung), Dermatitis (Hautentzündung), Nymphomanie (Hypersexualität bei Frauen), Polytoxikomanie (Abhängigkeit von Suchtstoffen), Trichotillomanie (Haarausreißsucht), Agoraphobie (Platzangst), Erythrophobie (übertriebene Errötungsfurcht), Klaustrophobie (Angst vor Aufenthalt in geschlossenen Räumen).

In der Gemeinsprache werden nach diesem Muster Wörter gebildet, wie Sammleritis, Cubanitis, Telefonitis, Zeltomanie, Pillomanie, Fußballmanie, Computer-Phobie, Bierphobie, Ansteckungsphobie.

Die Konstituente -itis in der Fachsprache hat die Bedeutung 'Entzündung', die Bedeutungsmerkmale wie 'krankhafter Zustand', 'akut' und 'entzündlich' enthält. 'Krankhaft' ist ein von der Norm abweichender Zustand, wobei als Norm 'die Gesundheit' zu sehen ist (vgl. Nortmeyer 1987: 395). In der Gemeinsprache bezeichnet -itis auch einen von der Norm abweichenden Zustand, wobei als Norm 'ein von der Gesellschaft erwartetes Verhalten' gemeint ist (vgl. Feine 2003). Wer an Sammleritis leidet, zeigt eine übermäßige Sammelaktivität, die von anderen Menschen als krankhaft empfunden wird, da sie von einer als Norm verstandenen Verhaltensweise abweicht. Ähnlich sind die Bildungen mit der Konstituente -manie zu verstehen. Fachsprachlich bezeichnet auch -manie einen krankhaften Zustand, der sich als zwanghaftes, maßloses Verlangen, als Gier nach etwas Bestimmtem äußert. Neue Wortbildungen mit -manie in der Gemeinsprache bezeichnen wie auch die -itis-Bildungen eine hyperaktive Verhaltensweise, die normabweichend empfunden wird, in Graduierungen zwischen krankhafter Leidenschaft und übertriebener Vorliebe für etwas.

Wer z. B. an Zeltomanie leidet, zeigt eine übertriebene Vorliebe für das Zelten oder für Zelte, wer an Pillomanie leidet, nimmt zu viele Tabletten ein. 
Vorsicht, Pillomanie! ${ }^{1}$ Soll man sich freuen oder erschrecken? Immer mehr Fragen des Lebens werden mit Tabletten entschieden! Per Pille kann man Kinder verhindern, aber - schmeck's, Fortschrittsfan! - nicht machen, dafür wäre eher Viagra zuständig. (Kleine Zeitung 1998)

Auch eine Phobie ist fachsprachlich verstanden eine abnorme, sich zwanghaft aufdrängende Angst als Form der Psychose (vgl. Roche-Lexikon Medizin (1993)). Die Phobiebildungen in der Gemeinsprache bezeichnen mentale Zustände von Menschen in Graduierungen zwischen 'Angst' und 'Abneigung', wobei nur der Kontext Aufschluss geben kann, welche Bedeutungsvariante gemeint ist:

Autophobie: Angst am Beifahrersitz Berlin. - Angstzustände beim Autofahren - daran leiden etwa fünf Prozent der Verkehrsteilnehmer. Davon sind vor allem Männer betroffen, die ihrer Ehefrau das Steuer überlassen. Die bissigen Kommentare über "ihre" Fahrkunst, die oft Anlaß für Ehekonflikte sind, stellen nur den Versuch der Männer dar, die eigene Furcht zu verdrängen. Darüber berichtet das deutsche Magazin "Auto/Straßenverkehr" und beruft sich auf eine Studie des Psychologen Rainer Schönhammer. (Neue Kronen-Zeitung 1996)

Vielleicht, weil man's nicht dekantieren kann (was heute ja zur Zeremonie jeder Einladung gehört); vielleicht, weil man nicht am Kronenkorken riechen kann (können schon, aber wie sieht das aus?); vielleicht aber auch, und das ist mein Verdacht, weil die Frauen überwiegend Bier nicht mögen. Sie sind ja gemeinhin der Maßstab allen guten Geschmacks, dem sich die Welt höflich beugt - selbst wenn sie so danebenhauen wie bei ihrer Einstellung zu Bier. Also, ich kenn' schon weiche, denen schmeckt's; aber ich kenn' mehr als die, die würden im Müllner-Beisel zu Salzburg einen G'spritzten bestellen. Auf dem amerikanischen Kontinent kennt man die weibliche Bierphobie auch, und diverse Brauereien wollen ihr zwecks Umsatzsteigerung jetzt zu Leibe rücken. Extra für Damen gebrauter Gerstensaft soll weniger herb und einen Spritzer exotischer sein. (Die Presse 1997)

In diese Reihe von -itis-, -manie- und -phobie-Bildungen in der Gemeinsprache passt auch die Fremdkonstituente -aholic/-oholic, obwohl sie eine andere Entstehungsgeschichte hat. Sie wurde erstmals in dem Lexem workaholic von dem amerikanischen Seelsorger Wayne Oates verwendet. Er verstand unter einem Workaholic „.... eine Person, die von ihrer Arbeit so abhängig ist wie andere vom Alkohol" (Angliszismen-Wörterbuch 1995: 1726). Das Lexem ist also eine Art Kontamination aus work und alkoholic und bezeichnet einen Menschen, der unter dem Zwang steht, ununterbrochen arbeiten zu müssen, was gesundheitliche Schäden nach sich zieht und im schlimmsten Fall zum Tode führen kann. Das Krankheitsbild selbst wird als

${ }^{1}$ Der Fettdruck der Fremdwortbildungen in allen Beispieltexten ist im Original nicht vorhanden. Er wurde eingefügt, damit die Bildungen schnell erfasst werden können. 
workaholism / Workaholismus bezeichnet. Die Konstituente -aholic löste sich aus dem Lexem Workaholic und wurde als Wortbildungselement (auch in der Variante -oholic) in der Gemeinsprache produktiv. Im Longman Dictionary of Contemporary English (1995: 28) wird sie als solches bereits aufgeführt: "-aholic: suffix ... someone who cannot stop doing something or using something: $a$ workaholic (= who loves working and cannot stop) $a$ chocaholic (= who loves chocolate)".

Neben dem Schokoholic werden in der Gemeinsprache Lexeme gebildet, wie Spielaholic, Aquaholik, Autoholic, Flamencoholiker, Internet-oholik, Sexaholic u. a.

-aholic/-oholic bezeichnet in solchen Bildungen eine Person mit einer von der gesellschaftlichen Norm abweichenden und deshalb häufig negativ gewerteten Verhaltenseigenschaft in graduellen Abstufungen zwischen Sucht (mit physischen, psychischen und finanziellen Folgeschäden) - das kann beim Spielaholic ohne weiteres der Fall sein - und Liebhaberei.

Im letzten Herbst las ich in einer Drogistenzeitung, dass vor allem Negaholiker von Grippeviren heimgesucht werden. Negaholiker sind, so stand es im Blatt, Menschen, die Böses tun und denken. Also Artgenossen, die süchtig nach Bösem sind. Gute Menschen bleiben gesund, hiess es. (Züricher Tagesanzeiger 1997)

Hätte uns bloss einmal jemand gesagt, er sei ein Geniesser gewesen, dieser Johann Wolfgang Goethe. Ein Schokoholic war er, Trinkschokolade liebte er über alles. Dem Frankenwein ... war er ebenso innig verbunden wie dem Champagner. Der Gänseleberpastete äusserst zugetan, aber auch den Würsten. (Züricher Tagesanzeiger 1999)

-itis-, -manie-, -phobie- und -aholic/oholic-Bildungen eignen sich auf Grund ihrer Semantik in besonderem Maße, das Verhalten von Menschen zu werten.

Da solche Bildungen häufig Ad-hoc-Bildungen sind, ist es unter textlinguistisch-pragmatischem Aspekt von Interesse, wie die neuen Wortbildungsprodukte im Text eingeführt und ob und welche Semantisierungshilfen gegeben werden. Oftmals werden sie im Text durch das graphische Mittel Anführungszeichen oder Doppelpunkt hervorgehoben:

Eine neue Eleganz hat die Damenmode des Sommers 2001 für sich ausgemacht ... Ein Revival von Reptilien favorisiert vor allem Schlangen- und Straußenoptiken. Zur neuen Lust auf Luxus passt eine gewisse „Logomanie“: Metallische Effekte und Gold unterstreichen die mondäne, elegante Wirkung. (Mannheimer Morgen 2001)

Das Volk hat dem Platz bereits seinen eigenen Namen gegeben: Protestodrom. (Die Zeit 2000)

Die Bedeutung der Wortneubildungen kann in vielen Fällen ohne zusätzliche Erläuterung vom Rezipienten entsprechend seinem Weltwissen aus 
dem Kontext erschlossen werden. Zuweilen werden Heckenausdrücke, wie eine Art von etw. sein, in der Art von etw. sein als Zuordnung zu einer Kategorie verwendet. Damit soll eventuell an die Bedeutung der fachsprachlichen Konstituente -itis, -manie oder -phob angeknüpft werden, diese jedoch durch den Heckenausdruck relativiert werden:

Eine Art "Ägyptomanie" hat die Öffentlichkeit erfasst und findet ihr Echo in einer wahren Buchflut. (Mannheimer Morgen 1999)

Hersteller von High-Tech-Produkten leiden an einer Art „Kunden-Phobie”. Die Unter-nehmensberatung Deloitte Touche kam bei einer Studie in Großbritannien zum Ergebnis, dass die Zufriedenheit der Kunden in den letzten Jahren immer weiter abgenommen hat, obwohl immer mehr Produkte auf den Markt kamen. (Die Presse 1998)

Werden spezielle Semantisierungshilfen gegeben, dann erfolgen sie in der Regel durch drei Verfahren: Es wird ein Synonym genannt, eine Definition gegeben oder durch Paraphrasierung erläutert.

\section{Synonym:}

Er unterzeichnete seine Briefe mit „Bourgeoisophobus“, der Bürgerfeind, um deutlich machen, wie sehr er die „dummen Kaufleute und ihresgleichen“ verachtete. (Die Zeit 2002)

Gegen das Vergessen drehte der Belgier Marc Hoogsteyns seinen Film „Kongomanie - Kongowahnsinn". (Mannheimer Morgen 2001)

Das Schöne in meiner Heimat ist: Die Maradonitis, das Maradona-Fieber, ist wieder da. (Salzburger Nachrichten 1994)

Schon haben sich erste Selbsthilfegruppen von „Webaholics” (Netz-Süchtigen) gebildet. (Neue Kronen-Zeitung 1995)

\section{Definition:}

Das Rollodrom ist ein Rollstuhllehrpfad, auf dem gesunde Menschen er"fahren" können, welche Hindernisse sich einem Rolli-Fahrer so täglich in den Weg stellen. (Vorarlberger Nachrichten 1999)

Berlusconitis ist, immer mit telegenem Anzug und telegener Krawatte herumzulaufen, immer sonnengebräunt und geschminkt mit allen 42 Zähnen zu lächeln ... auf die Fragen nicht zu antworten, sondern stur an der eingepaukten Story festzuhalten. (Oberösterreichische Nachrichten 1996)

"Shopaholics" sind iene, für die die Schnäppchenjagd zum Lebensinhalt wird, die sich von seelischem Leid freikaufen durch flotte Kleider, neues Geschirr oder wirk- 
lich unbrauchbare Dinge wie Hunderte Flaschenöffner oder Mixer. (Salzburger Nachrichten 1996)

Paraphrase:

Von Artomanie (übersteigerte Beschäftigung mit der Kunst) und Augenschwäche ... handelt das Buch. (Mannheimer Morgen 2000)

Warum das so ist, weiß niemand genau; doch in dieser Lebensspanne, soviel steht fest, gerät so mancher von uns unter das erbarmungslose Joch der Autogerontophobie, der Angst vor dem eigenen Altern. (TAZ 1991)

Sie ist vielmehr von einer Krankheit befallen, der "Scheusaleritis", sieht sich ständig von einem grünen Scheusal verfolgt und rennt schreiend und tobend durch die Räume. (Mannheimer Morgen 1989)

Unter textlinguistisch-pragmatischem Aspekt ist weiterhin von Interesse, welche Funktionen solche Bildungen in welchen Arten von Texten erfüllen.

Eine sprachökonomische Funktion können sie aufgrund ihrer Struktur erfüllen. Wie schon festgestellt, bringt die zweite Konstituente - -itis und -manie - ganz allgemein eine von der gesellschaftlichen Norm abweichende und deshalb häufig negativ gewertete Verhaltenseigenschaft in graduellen Abstufungen zwischen Sucht und Liebhaberei zum Ausdruck bzw. -aholic eine Person, die eine solche Verhaltensweise zeigt.

Die erste Konstituente gibt entweder die spezifische Tätigkeit an, die in einem Übermaß ausgeführt wird, oder ein Objekt im weitesten Sinne, auf welches das entsprechende Verhalten gerichtet ist. Dabei kann die erste Konstituente auch eine Person bezeichnen:

Sammelitis / Sammelmanie - eine übermäßige Sammeltätigkeit;

Collectoholic - von einer Sammelleidenschaft besessener Mensch;

Musicalitis/Musicalmanie - eine abnorme Leidenschaft für Musicals;

Musicalaholic - von einer Leidenschaft für Musicals besessener Mensch;

Genscheritis, Gorbi-Manie, Goethaholic - von einer Leidenschaft für Genscher, Gorbatschow, Goethe besessen sein.

Bei Phobie-Bildungen sind die ersten Konstituenten auch tätigkeits- oder objektfokussiert in dem Sinne, dass sie die Tätigkeiten oder Objekte bezeichnen, vor denen die krankhafte Angst besteht bzw. auf welche die übermäßig starke Abneigung gerichtet ist:

Fahrphobie - krankhafte Angst / Abneigung zu fahren;

Hundephobie - krankhafte Angst vor Hunden / Abneigung gegen Hunde.

Auch hier kann die erste Konstituente eine Person bezeichnen:

Haider-Phobie - Abneigung gegen Haider. 
Dadurch, dass die Spezifik des Verhaltens und Wertung in einem Wort komprimiert sind, erfüllen sie eine sprachökonomische Funktion.

Hinzu kommt ein weiterer Aspekt: Die meisten dieser neuen Wortbildungsprodukte sind Okkasionalismen, und nur wenige werden lexikalisiert. Durch ihre Neuheit und Andersartigkeit wirken sie expressiv, erregen die Aufmerksamkeit der Rezipienten und schaffen Leseanreiz.

Die Quellen der bisher zitierten Textbeispiele zeigen, dass solche Wortneubildungen hauptsächlich in Pressetexten auftreten. Sie scheinen typisch zu sein für die Textklasse der medialen Kommunikation, die informierende, meinungsbildende, appellierende $u$. a. Sorten von Texten umfasst. Leider lassen sich die Textbeispiele, die dem Textkorpus COSMAS II des Instituts für deutsche Sprache in Mannheim entnommen sind, derzeit auf Grund des beschränkten Kontextes nur bedingt zuordnen. Sie sind überwiegend in informations- und meinungsbetonten Textsorten zu finden und werden häufig in humoristischen und ironisierenden Kontexten bewusst als verstärkendes Stilmittel genutzt:

Die „accelerierte Monsteritis" Nun ist es ja so, daß in Kindern oft der Wunsch entsteht, den elterlichen Haushalt mit einem Tier zu vervollkommnen. Das wäre nicht weiter tragisch, wenn dadurch nicht eine fatale Entwicklung Platz greifen würde, die Familienforscher mit Furcht in der Stimme als „accelerierte Monsteritis" bezeichnen. Im Klartext heißt das: Je näher die Pubertät rückt, desto hartnäckiger wird der Haustierwusch verfolgt und desto monströser fällt er aus.

Buben infizieren sich mit "Monsteritis" üblicherweise an Fischen: Sechs- bis Achtjährige geben sich noch mit Neonsalmern und ähnlichen Schwanzerln im Aquarium zufrieden. Kaum der Volksschule entwachsen, steigt die Untergrenze des tierischen Prestige in Richtung Piranhaschwarm. Männliche Mittelschüler halten Vogelspinnen für den Inbegriff der Putzigkeit und Tigerphytons für echt megageil. (Oberösterreichische Nachrichten 1997)

Dieter Bohlen und Ralph Siegel, Komponisten, liefern sich weiter einen heftigen Schlagabtausch. Nachdem Bohlen dem Konkurrenten zum Rückzug ins Privatleben geraten hatte, sagte der Angegriffene in einem Interview: „Herr Bohlen hat wohl seit langem eine Siegel-Phobie - vielleicht sollte der mal einen Psychiater konsultieren.” Bohlen hatte vor Kameras gesagt: „Warum soll ich Siegel zum Sieg gratulieren? Ich finde das Lied Sch..." (Mannheimer Morgen 2002)

Damit wird noch ein weiterer Aspekt untersuchenswert, und zwar der der semantischen Konkurrenzen und Konvergenzen zwischen den exogenen Einheiten, aber auch zwischen exogenen und indigenen Elementen. Liegt Bedeutungsäquivalenz zwischen den Elementen vor, sind sie also synonym, führt das dazu, dass sie im Sprachgebrauch konkurrieren. Allerdings kommt eine totale Äquivalenz sehr selten vor. Es würde bedeuten, dass Wortbil- 
dungen mit solchen Elementen sowohl im denotativen als auch im konnotativen Bedeutungsbereich übereinstimmen und in jedem Kontext substituierbar wären. Das allerdings würde dem Prinzip der Sprachökonomie widersprechen. Meist ist es so, dass die Bedeutungen im denotativen Bereich eine weitaus größere Übereinstimmung zeigen als im konnotativen Bereich. Man fasst den Begriff der Syonymie in der Regel nicht so eng. Bußmann (1990) spricht z. B. von „Bedeutungsgleichheit (bzw. Bedeutungsähnlichkeit) von zwei oder mehreren sprachlichen Ausdrücken". Im Sprachgebrauch ist auch zu beobachten, dass bestimmte Ausdrücke aus verschiedenen Gründen synonym verwendet werden (z. B. Keltenmanie - Keltenwahn, Rattenphobie - Rattenangst) und damit konkurrieren, obwohl keine totale Bedeutungsäquivalenz vorliegt. Die feinen Differenzierungen im konnotativen Bereich sind schwer erfassbar und mehr oder weniger von der Intuition des Produzenten bzw. Rezipienten abhängig. So kann es ohne weiteres sein, dass mehrere Ausdrücke, die zu demselben Paradigma gehören, zwar in bestimmten semantischen Merkmalen übereinstimmen, sich also mehr oder weniger ähnlich sein können, aber nicht völlig identisch sind. Diesen Sachverhalt bezeichnet man auch als semantische Konvergenz, die dem Sprachbenutzer eine Auswahl aus alternativen Ausdrücken ermöglicht. Bei Erben (1993: 37 f.) heißt es dazu:

„Da nicht selten eine Konvergenz zwischen verschiedenen wortbildenden Morphemen besteht, die im gleichen Wortbildungsparadigma (Funktionsstand) zusammenwirken, hat der Sprecher bis zu einem gewissen Grade auch die Wahl zwischen Affixen, die ähnliches leisten, ja z. T. sogar als Konkurrenten mit der gleichen Basis verbunden werden können und im gleichen Kontext ohne Informationsunterschied austauschbar sind. ... Auf der anderen Seite ist deutlich, daß nicht jedes Morphem mit jedem beliebigen anderen willkürlich verbunden werden kann. Es gibt Einschränkungen, die man herausfinden und möglichst regelhaft (Restriktionsregeln) beschreiben muß, ebenso wie die Affinitäten (Bindungsneigungen) bestimmter Affixe zu Morphemen bestimmter Basisklassen ...".

Es wurde schon festgestellt, dass eine Bedeutungsähnlichkeit zwischen -itis- und manie-Bildungen besteht, da beide eine hyperaktive, normabweichende Verhaltensweise in Graduierungen zwischen 'krankhaft' und 'übermäßig stark' beinhalten. Es zeigt sich auch, dass beide Fremdelemente mit der gleichen ersten Konstituente verbunden werden und demzufolge als Konkurrenten auftreten können.

Die folgende Übersicht zeigt solche aufgetretenen Parallelbildungen.

\begin{tabular}{|l|l|}
\hline Abkürzitis & Abkürzungsmanie \\
\hline Anwaltitis & Anwaltsmanie \\
\hline Computeritis & Computer-Manie \\
\hline
\end{tabular}




\begin{tabular}{|l|l|}
\hline Cubanitis & Kubamanie / Kuba-Manie \\
\hline Eventitis & Eventomanie / Event-Manie \\
\hline Festivalitis & Festivalmanie \\
\hline Fusionitis & Fusionsmanie / Fusionierungs-Manie \\
\hline Gipfelitis & Gipfelmanie / Gipfel-Manie \\
\hline Handyritis & Handymanie / Handy-Manie \\
\hline Konzeptionitis & Konzept-Manie \\
\hline Maradonitis & Maradona-Manie \\
\hline Marathonitis & Marathonmanie \\
\hline Monsteritis & Monster-Manie \\
\hline Musicalitis & Musical-Manie \\
\hline Natelitis & Natel-Manie \\
\hline Oranjitis & "Oranje"-Manie \\
\hline Polleritis & Poller-Manie \\
\hline Quizeritis & Quizmanie \\
\hline Sammleritis & Sammelmanie / Sammel-Manie \\
\hline Sanktionitis & Sanktionsmanie \\
\hline Telefonitis & Telefonmanie / Telefoniermanie \\
\hline Zitatitis & Zitiermanie \\
\hline
\end{tabular}

Es entsteht die Frage, ob diese Bildungen in Texten synonym verwendet werden und der Ausdrucksvariabilität dienen oder ob sie inhaltliche Nuancierungen zum Ausdruck bringen und semantische Nischen füllen. Es kann hier keine erschöpfende Antwort auf diese und im Weiteren noch entstehende Fragen gegeben werden, es soll lediglich gezeigt werden, welchen Aspekten in weiteren Forschungen noch nachgegangen werden muss.

Einige Beispiele zum Vergleich:

(1a) Computer-Manie verriet den Flughafen-Erpresser

Seine Computer-Manie verriet einen 25jährigen, der 15 deutschen Flughäfen gedroht hatte, durch ein ferngesteuertes Modell ein Passagierflugzeug abstürzen zu lassen. (Neue Kronen-Zeitung 1997)

(1b) Computer-Lernboom unter der Lehrerschaft

Unter steirischen Lehrem ist rekordverdächtige Lernlust, vor allem aber heftige Computer-Neugier ausgebrochen. In den ersten und in der letzten Ferienwoche geht die Lehrerschaft im Lande traditionsgemäß auf Fortbildungstrip. Speziell fällt auf, daß nun offenbar auch unter der Lehrerschaft an Volksschulen die "Computeritis" ausgebrochen ist - vor allem Einsteigerkenntnisse sind gefragt. (Kleine Zeitung 1997)

(2a) Das Megaevent

Sind steirische Politiker in den vergangenen Jahren mit Ankündigungsparolen leergelaufen, so regiert nun die "Eventomanie". Millionen Steuergelder werden für "Events" vergeudet, denen es an Nachhaltigkeit fehlt und die jederzeit austauschbar sind. (Kleine Zeitung 1998)

(2b) Doch jetzt beglückt uns die Eventitis mit einer neuen Form - dem BranchenCrossover. Das Zürcher Möbelgeschäft Rosenberger und Hollinger zum Beispiel tut sich für eine Erlebniswoche mit Moët \& Chandon zusammen, um neue Kunden zu 
gewinnen und die alten bei Laune zu halten. In Erlenbach zeigt Reiseveranstalter Kuoni seine schönsten Destinationen zu Mode von Robert Ober, als Dritter im Bunde serviert Traiteur Oggenfuss Cocktailhäppchen. Was soll dieser Gemischtwarenhandel? (Züricher Tagesanzeiger 1999)

(3a) Fiebrige Gipfelitis

G-8 steht längst als Synonym für die weltweit zu beobachtende "Gipfelitis", das fiebrige Streben nach immer aufwendigeren, weil besser vermarktbaren Treffen auf höchster Ebene. Auf den Inhalt kommt es immer öfter gar nicht an. Übertrieben? Vielleicht, aber Pomp und Erwartung stehen in keiner Relation zu dem, was an Substanz allenfalls bleibt. (Die Presse 2000)

(3b) Aber noch ist kein Ende der Gipfelmanie in Sicht. Vorbei die Zeit, wo "Gipfel" fürs Treffen Reagan-Gorbatschow reserviert war. Vor uns die galoppierende GipfelInflation, die selbst das Plauscherl zwischen Hausmeisterin und Untermieter zum „Wohnungsgipfel" aufplustern wird. (Die Presse 1994).

(4a) Kubanische Künstler profitieren von der „Cubanitis" und einem Tauwetter zwischen Washington und Havanna

... Gleichzeitig kommt den Inselmusikern ein Phänomen zugute, das Insider schon "Cubanitis" tauften. Ein Virus, der nicht nur Miamis Musikszene bestimmt. Kuba ist in, und die Zeichen sind unübersehbar. Sei es, daß Schauspieler wie Jack Nicholson und Leonardo Di Caprio auf der Insel gesichtet werden und ihren Aufenthalt sichtlich genießen, daß Kuba-Fans sich mit Perlenkettchen der afrokubanischen Religion Santeria schmücken, US-Amerikaner nach kubanischen Zigarren gieren und in SalsaKursen die Hüften schwingen. (Frankfurter Rundschau 1998)

(4b) Bereits 1991, also lange vor Einsetzen der musikalischen Kuba-Manie, veröffentlichte die kanadische Sopransaxophonistin, Flötistin und Bandleaderin Jane Bunnett ihr von der vielfältigen Musik Kubas beeinflusstes Album „Spirits Of Havanna“. (Musik Newsletter, Internet, musik-news.de)

Im Beispiel (1a) ist m. E. Computer-Manie nicht durch Computeritis ersetzbar. Text (1a) ist in der Art einer Kurznachricht verfasst und hat dadurch einen ernsthafteren Charakter als Text (1b), der eine mehr scherzhafte Grundhaltung aufweist. Umgekehrt ist im Text (1b) auch Computeritis nicht durch Computer-Manie ersetzbar. Das liegt daran, dass -itis- und -manieBildungen in ihrer Semstruktur neben gleichen Semen auch unterschiedliche haben, die bereits in der fachsprachlichen Bedeutung von -itis und Manie angelegt sind und in die gemeinsprachliche Bedeutung mit eingehen. Manie beinhaltet das Sem 'chronisch', also 'sich langsam entwickelnd' und 'lange oder ständig anhaltend'. Eine Manie ist ein anhaltendes, unter Umständen unheilbares Leiden. Dadurch wird sie als eine ernsthaftere Krankheit empfunden als eine "-itis", also eine Entzündung, die im Gegensatz zur Manie meist akut ist, also unvermittelt auftritt, schnell und heftig verläuft, aber meist heilbar ist. -itis-Bildungen in der Gemeinsprache beinhalten das Sem 'akut'. Eine "Computeritis" kann deshalb auch plötzlich ausbrechen, wie im 
Text (1b) dargestellt. Eine "Computer-Manie" würde langsam entstehen und wäre mit "ausbrechen" im Text (1b) nicht kompatibel.

In ähnlicher Weise können auch die übrigen Beispiele betrachtet werden. Eine Substitution der -manie-Bildungen durch die -itis-Bildungen und umgekehrt ist nicht $\mathrm{zu}$ empfehlen. Bestimmte Kontextelemente der -itis- und -manie-Bildungen aktualisieren die obengenannten Seme von -itis und -manie und lassen deshalb die gewählte -itis- bzw. -manie-Variante als die angemessenere erscheinen. So tritt z. B. "Glück" im Leben meist plötzlich und unerwartet auf, deshalb ist Eventitis in (2b) eher mit beglücken in Verbindung zu bringen als Eventomanie.

$\mathrm{Da}$ Fieber eine häufige Begleiterscheinung von Entzündungen ist, schwingt auch in den -itis-Bildungen in der Gemeinsprache das Sem , fieberhaft' mit. In (3a) ist daher die Kombination von "fiebrige Gipfelitis" angemessen, eine Substitution von Gipfelitis durch Gipfelmanie nicht.

Ein Virus, das sich epidemiehaft schnell verbreitet ist wie in (4a) besser mit Cubanitis verbindbar als mit Kuba-Manie.

Die unterschiedlichen Krankheitsbilder von einer Manie und einer "-itis", die in den gemeinsprachlichen -itis- und -manie-Bildungen mitschwingen, bestimmen wahrscheinlich ihren bevorzugten Gebrauch in bestimmten Textsorten. Es hat den Anschein, dass -itis-Bildungen verwendet werden, wenn der Text eine scherzhaft-spöttische Grundhaltung hat, während -manieBildungen in Texten mit einer ernsthafteren, kritisch-ironischen Grundhaltung auftreten.

Bisher wurden Parallelbildungen mit Fremdelementen betrachtet. Es können aber auch Derivate mit indigenen Affixen, deren Basiskonstituente vergleichbar ist mit tätigkeitsfokussierten -itis- und/oder -manie-Bildungen unter dem Aspekt der Konvergenz bzw. Konkurrenz untersucht werden. Dabei geht es in erster Linie um das Suffix -ei mit den Suffixvarianten -erei/ -elei und um das Zirkumfix Ge-...-e. Die Suffixvarianten -erei/-elei haben sich nach Fleischer/Barz (vgl. 1992: 149f.) aus Suffixbildungen mit -ei nach Mustern wie Eselei, Ferkelei, Kantorei, Kinderei entwickelt: Fremdwörtelei, Lumperei. Sie weisen vorwiegend eine pejorative Konnotation auf. -erei-Bildungen werden - als pejorative Prozessbezeichnung - auch von einer verbalen Basis abgeleitet, wobei -er- meist nicht zum Verbstamm gehört: Schreierei, Esserei. Von Verben auf $-(e) l-n$ werden Prozessbezeichnungen wie Blödelei, Faselei abgeleitet, wobei nach Fleischer/Barz (1992: 150) solche Bildungen „weniger pejorativ, sondern eher salopp-scherzhaft" sind.

Die von Verben abgeleiteten -erei-/-elei-Bildungen stehen in Konkurrenz zu den Deverbativa mit dem Zirkumfix Ge-...e: Geschreibe. Auch sie sind pejorativ konnotiert und enthalten das Merkmal des Überdrüssigen (vgl. Fleischer/Barz 1992: 208). 
In Texten wurden dazu folgende Wortbildungen gefunden:

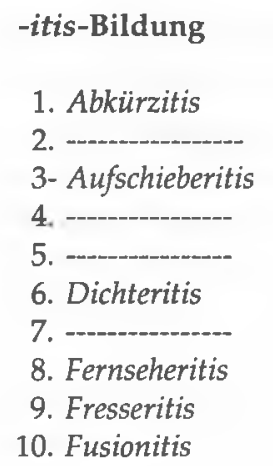

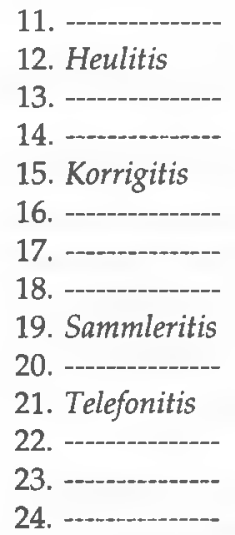

-manie-Bildung

Abkürzungsmanie

Aufräummanie

Baumanie

Befragungsmanie

Einkaufsmanie

Fusionsmanie /

Geheimhaltungsmanie

Kontrollmanie

Kopiermanie

Putzmanie

Redemanie

Reisemanie

Sammelmanie

Tauschmanie

Telefoniermanie /

Verpackungsmanie

Wegwerfmanie

Zählmanie
-erei/-elei-Bildung

Ge...-e-Bildungen

Aufräumerei

Aufschieberei

Bauerei

Befragerei

Dichterei

Einkauferei

Fernseherei

Fresserei

Fusioniererei

Fusionierungsmanie

Geheimhalterei

Heulerei

Kontrolliererei

Kopiererei

Korrigiererei

Putzerei

Rederei

Reiserei

Sammelei / Sammlerei

Tauscherei

Telefoniererei

Verpackerei

Wegwerferei

Zählerei

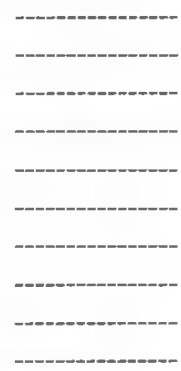

Auffällig bei den -manie-Bildungen ist, dass die erste Konstituente nicht immer ein Verb ist wie bei den -itis-, -erei- und Ge...-e-Bildungen, sondern ein Deverbativum auf -ung: Abkürzungs-, Befragungs-, Geheimhaltungsmanie usw.

Auch bei diesen Bildungen ist zu untersuchen, inwieweit Konkurrenz bzw. Konvergenz zwischen ihnen besteht.

Einige Beispiele:

(5a) „Fusionitis" erfasst die Westschweizer Politiker. Der Globalisierungs-Erdrutsch und die dadurch ausgelöste Konzentrationswelle beschränken sich nicht auf kommerzielle Aktivitäten, den Dienstleistungssektor und die Industrie. Armee, Sport, Gesundheit, Kultur und Medien sind ebenfalls vom Restrukturierungsvirus erfasst worden. (St. Galler Tagblatt 1998)

(5b) Die Rezepte der Führung vermochten bisher wenig. Der noch vom 15. Parteitag im Herbst 1997 propagierte Zusammenschluss desolater Unternehmen mit gesunden Einheiten hat sich vielerorts als Flop erwiesen: Zahlreiche zunächst solvente Betriebe sind von den verschuldeten mit in den Abgrund gerissen worden. Jetzt wettert die Pekinger "Volkszeitung" gegen die Fusionsmanie. (Die Presse 1998) 
(5c) Der oben genannte Artikel in bezug auf die Fusionierungsmanie ist ganz geprägt von der Einsicht, der Ohnmacht und der Hilflosigkeit, dem wir als „Volk“ gegenüberstehen. Die Profitsucht der "Großen“ ist gepaart mit Ignoranz und Kurzsichtigkeit. ... Unternehmen, die heute hochgelobt und als beispielgebend hingestellt werden, sind in Wahrheit menschenverachtend. (Oberösterreichische Nachrichten 1998)

(5d) "Grundsätzlich legen wir aber Wert auf klein- und mittelständische Strukturen." Darauf pocht auch Manfred Holztrattner, Chef des Salzburger Raiffeisenverbandes und damit Miteigentümer. Das Dach des Techno-Z biete die Möglichkeit, daß sich Unternehmen selbständig entwickeln, gleichzeitig aber kooperieren und gemeinsam Infrastruktur nutzen können. "Das ist ein Ausweg aus der ständigen Fusioniererei", so Holztrattner. (Salzburger Nachrichten 1998)

(6a) Das Ehepaar Heidekorn leidet unheilbar an schwerer Sammleritis. Im Häuschen an der Nidda gibt es Gerippte in verschiedensten Variationen sowie exotisch frankierte Rödelheim-Kuverts. ... Im Laufe der Jahre hat das "sammelverrückte Paar", wie Freunde liebevoll sagen, das idyllische Häuschen in ein kleines Museum verwandelt. (Frankfurter Rundschau 1998)

(6b) Im Gegensatz zur Staatsanwaltschaft, die für Schneider sieben Jahre und neun Monate Freiheitsentzug gefordert hatte, kam das Gericht nach 41 Verhandlungstagen zu dem Ergebnis, daß Schneider vor den Banken nicht als „abgebrühter Großbetrüger" aufgetreten sei. Vielmehr sei der eher biedere Ingenieur einer "glaubhaften Obsession für alte schöne Häuser erlegen, die er in einer Art Sammelmanie behalten wollte, obwohl es sich wirtschaftlich nicht rechnete", sagte Vorsitzender Richter Heinrich Gehrke in der Urteilsbegründung. (Frankfurter Rundschau 1997)

(6c) Damals haben drei Mitarbeiter der städtischen Gemeindeverwaltung drei Tage lang seine Engelscharen für das Guinnessbuch gezählt. Das tut die Stadt gerne, man ist schließlich stolz auf Herrn Fischer. Der Mann ist eine Attraktion für den Ort, außerdem hat er mit dem Rekord einen Sammler aus den USA übertrumpft. Nun gibt es Menschen, die ihn wegen seiner Sammlerei merkwürdig finden - doch das stört ihn nicht. (Mannheimer Morgen 2002)

(7a) Digital-Funken - Mobiltelefon auf dem Vormarsch

In Italien gehört es zum - geschäftlich wie privat - Alltag, ob im Auto oder auf der Vespa: das "Handy". In Deutschland hat die "mobile Telefonitis" längst noch nicht die Dimensionen angenommen wie in anderen europäischen Ländern. Doch der Funkenflug ist nicht mehr zu löschen. (Mannheimer Morgen 1994)

(7b) In keiner anderen Stadt der Erde gibt es gemessen an der Bevölkerung so viele tragbare Telefone. Etwa jeder vierte Hongkonger über 15 Jahren besitzt ein Handy. Allerdings hat die Telefoniermanie auch ihre Schattenseiten. (Oberösterreichische Nachrichten 1997)

(7c) Obwohl niemand genau weiss, ob das Telefonieren mit dem Handy wirklich gefährlich ist, möchte die Erdövereinigung mit einem Handy-Verbot-Kleber der Telefoniererei ein Ende bereiten, zumindest bei Tankstellen. (St. Galler Tagblatt 1999)

(8a) Ein ganz normales Familienleben ist, seitdem wir im November 1997 von den Schadstoffbelastungen erfahren haben, überhaupt nicht mehr möglich, da die Angst vor einer drohenden Gesundheitsgefährdung, eine mittlerweile schon auffällige Putzmanie, ständige Auseinandersetzungen mit den Kindern, die doch auf dem Boden 
spielen, sowie eine regelmäßige Mitarbeit in der Schadstoffinitiative dies nicht mehr zulassen. (Frankfurter Rundschau 1998)

(8b) Werner Hinze knallte die Bierdose auf den Tisch, daß der Schaum nur so spritzte. „Dein Geputze, dein Gejammer, dein Herumgemecker ... Ich habe den Kanal gestrichen voll!" (Oberösterreichische Nachrichten 1996)

Gemeinsam ist all diesen Wortbildungen, dass sie ein sprachliches Mittel der Wertung darstellen. Sie werden verwendet, um bestimmte Erscheinungen bzw. Verhaltensweisen von Menschen zu kritisieren. Sie unterscheiden sich darin, in welcher Art und Weise die Kritik zum Ausdruck gebracht wird. Daran ist auch die Schärfe der Kritik ablesbar - sie reicht von einer leichten über eine ernsthafte bis zu einer verletzenden. Das ist aber nicht allein nur an den Wortbildungen messbar, sondern hängt in mehr oder weniger starkem Maße auch von den Kontextelementen ab.

Für die -itis- und -manie-Bildungen in den Beispielen (5) bis (7) trifft das $\mathrm{zu}$, was schon bei der Darstellung der Beispiele (1) bis (4) gesagt wurde. Durch -itis-Bildungen und die entsprechenden Kontextelemente wird eine leichte Kritik auf eine mehr scherzhafte Art und Weise zum Ausdruck gebracht: Die Fusionitis erfasst (5a) wie eine Epidemie breite Kreise und breitet sich wie ein Funkenflug (7a) aus. Auch wenn in (6a) zunächst von einer schweren, unheilbaren Sammleritis die Rede ist, wird durch die folgenden Textelemente, wie $z$. B. das "sammelverrückte Paar", wie Freunde liebevoll sagen, die Scherzhaftigkeit der Aussage deutlich. In den Texten mit -manieBildungen ist eine ernsthaftere, schärfere Kritik spürbar, wobei Fusionsmanie (5b) und Fusionierungsmanie (5c) nahezu bedeutungsäquivalent sind und als Konkurrenten auftreten können. In (6b) wird durch den Heckenausdruck in einer Art auf die fachsprachliche Bedeutung von Manie Bezug genommen und damit auf eine Krankheit, wodurch die Schuldhaftigkeit der Handlungsweise Schneiders gemildert werden soll.

Die -erei-Bildungen (5d), (6c), (7c) haben zwar keine Bedeutungselemente, die einen Bezug auf eine leichte oder ernste krankhafte Verhaltensweise zulassen, besitzen aber das Merkmal 'pejorativ', also ,negativ wertend'. Dabei gibt die Basiskonstituente die Art der Handlung an und das Suffix -erei die Merkmale 'dauerhaft' und 'lästig', wodurch zum Ausdruck gebracht wird, dass eine Handlung von jmdm. so lange ausgeführt wird, dass sie von anderen als störend, unangenehm, in mehr oder weniger starkem Maße aufdringlich empfunden wird. Diese Merkmale treffen auch auf die Zirkumfixbildungen mit Ge...e- zu, so dass die beiden Bildungen als Konkurrenten auftreten. Die Zirkumfixderivate mögen durch ihre etwas aufwändigere Bildungsart noch nachdrücklicher wirken. In einem entsprechenden Kontext können sie eine verletzende Kritik bewirken (8b).

Der hier nur ansatzweise vorgenommene Vergleich zwischen Wortbildungen mit exogenen und solchen mit indigenen Elementen lässt zwar einen gewissen Konvergenzbereich erkennen, macht aber auch deutlich, dass jedes 
Element spezifische Merkmale in sich trägt, die es einzigartig machen. Die fremden Einheiten sind in der Lage, semantische Nischen auszufüllen, für die heimische Morpheme nicht zur Verfügung stehen.

Die angerissenen Untersuchungsaspekte zu exogenen Einheiten in der Wortbildung machen deutlich, dass die Fremdwortbildungsforschung noch am Anfang steht und eine ganze Reihe von Fragen erst durch detaillierte Analysen zu klären sind.

\section{BIBLIOGRAPHIE}

Altmann, Hans; Kemmerling, Silke, 2000. Wortbildung fürs Examen. Studien- und Arbeitsbuch. - Wiesbaden: Westdeutscher Verlag.

Bußmann, Hadumod, 1990. Lexikon der Sprachwissenschaft. Stuttgart: Kröner.

Cottez, Henri, 1989. Dictionnaire des structures du vocabulaire savant. Éléments et modèles de formation. Paris: Robert.

Donalies, Elke, 2000. Das Konfix. Zur Definition einer zentralen Einheit der deutschen Wortbildung. In: Deutsche Sprache 2: 144-159.

Donalies, Elke, 2002. Die Wortbildung des Deutschen. Ein Überblick. Tübingen: Narr (Studien zur deutschen Sprache 27).

Erben, Johannes, 1993. Einführung in die deutsche Wortbildungslehre. Berlin: Schmidt.

Erben, Johannes, 2000. Einführung in die deutsche Wortbildungslehre. Berlin: Schmidt.

Feine, Angelika, 2003. Fußballitis, Handyritis, Chamäleonitis -itis-Kombinationen in der deutschen Gegenwartssprache. In: Sprachwissenschaft 28 4: 437-466.

Feine, Angelika, 2005. Habermann, Mechthild: Eine korpusgestützte Analyse zur deutschen Fremd-Wortbildung. Möglichkeiten und Grenzen. In: Schwitalla, Johannes; Wegstein, Werner (Hg.): Korpuslinguistik deutsch: synchron - diachron - kontrastiv. Würzburger Kolloquium 2003. - Tübingen: Niemeyer: 89-98.

Fischer, Eberhard, 1985. Das „gebundene Grundmorphem“ in der deutschen Sprache der Gegenwart. In: Beiträge zur Erforschung der deutschen Sprache 5: 210-224.

Fleischer, Wolfgang: Konfixe, 1995. In: Wort und Wortschatz. Beiträge zur Lexikologie. Inge Pohl und Horst Ehrhardt (Hg.). Tübingen: Niemeyer: 61-68.

Fleischer, Wolfgang; Barz, Irmhild, 1995. Wortbildung der deutschen Gegenwartssprache. - Tübingen: Niemeyer.

Grimm, Hans-Jürgen, 1997. Konfixe: Beobachtungen in Tageszeitungen und in Wörterbüchern. In: Barz, Irmhild; Schröder, Marianne (Hg.): Nominationsforschung im Deutschen. Festschrift für Wolfgang Fleischer zum 75. Geburtstag. Frankfurt am Main u. a.: Lang: 277-284

Hoppe, Gabriele et al., 1987. Deutsche Lehnwortbildung. Beiträge zur Erforschung der Wortbildung mit entlehnten WB-Einheiten im Deutschen. Tübingen: Narr.

Hoppe, Gabriele: Herausbildung und Integration des Submusters ETHNIKA + -(o)phonie im Französischen und Stellung des analogen Musters im Lehn-Wortbildungssystem des Deutschen. - amades (Arbeitspapiere und Materialien zur deutschen Sprache). Hrsg. vom Institut für Deutsche Sprache) Nr. 0.

Hoppe, Gabriele, 1999. Das Präfix ex-. Beiträge zur Lehn-Wortbildung. Tübingen: Narr, (Studien zur deutschen Sprache 15).

Hoppe, Gabriele, 2000. Aspekte von Entlehnung und Lehn-Wortbildung am Beispiel (o)thek. - amades (Arbeitspapiere und Materialien zur deutschen Sprache), Hg. vom Institut für Deutsche Sprache) Nr. 1. 
Kinne, Michael, 2000. Die Präfixe post-, prä- und neo-. Beiträge zur Lehn-Wortbildung. Tübingen: Narr (Studien zur deutschen Sprache 18).

Kirkness, Alan, 1996. Zur lexikographischen Dokumentation eurolateinischer Wortbildungseinheiten: Vergleichende Beobachtungen am Beispiel aero-. In: Munske, Horst Haider; Kirkness, Alan (Hg.): Eurolatein. Das griechische und lateinische Erbe in den europäischen Sprachen (Reihe Germanistische Linguistik 169). Tübingen: Niemeyer: 236-274.

Klosa, Annette, 1996. Negierende Lehnpräfixe des Gegenwartsdeutschen. Heidelberg: Winter (Germanistische Bibliothek, 3. Reihe, Bd. 22).

Kocourek, Rostislav, 1982 La langue française de la technique et de la science. Vers une linguistique de la langue savante. Wiesbaden: Brandstetter.

Longman Dictionary of Contemporary Englisch. Langenscheidt-Longman, 1995.

Martinet, André, 1980. Éléments de linguistique générale. Nouvelle édition remaniée et revue, avec compléments et bibliographie. Paris: Colin.

Müller, Peter O., 2000. Deutsche Fremdwortbildung. Probleme der Analyse und der Kategorisierung. In: Habermann, Mechthild; Müller, Peter O.; Naumann, Bernd (Hg.): Wortschatz und Orthographie in Geschichte und Gegenwart: Festschrift für Horst Haider Munske zum 65. Geburtstag. Tübingen: Niemeyer: 115-134.

Müller, Peter O. (Hg.), 2005 Fremdwortbildung. Theorie und Praxis in Geschichte und Gegenwart. Frankfurt am Main: Lang.

Nortmeyer, Isolde, 1987. Untersuchung eines fachsprachlichen Lehnwortbildungsmusters: -itis-Kombinatorik in der Fachsprache der Medizin. In: Hoppe, Gabriele et al.: Deutsche Lehnwortbildung. Beiträge zur Erforschung der Wortbildung mit entlehnten WB-Einheiten im Deutschen. Tübingen: Narr: 331-408.

Nortmeyer, Isolde, 2000. Die Präfixe inter- und trans-. Beiträge zur Lehn-Wortbildung. Tübingen: Narr (Studien zur deutschen Sprache 19).

Ruf, Birgit, 1996: Augmentativbildungen mit Lehnpräfixen. Eine Untersuchung zur Wortbildung der deutschen Gegenwartssprache. Heidelberg: Winter (Germanistische Bibliothek, 3. Reihe, Bd. 25).

Schmidt, Günter Dietrich, 1987. Das Kombinem: Vorschläge zur Erweiterung des Begriffsfeldes und der Terminologie für den Bereich der Lehnwortbildung. In: Hoppe, Gabriele: Deutsche Lehnwortbildung. Beiträge zur Erforschung der Wortbildung mit entlehnten WB-Einheiten im Deutschen. Tübingen: Narr: 37-52.

Schmidt, Günter Dietrich, 1980. Therm(o). Untersuchungen zu Morphosyntax, Geschichte, Semantik und anderen Aspekten einer produktiven LWB-Einheit im heutigen Deutsch. In: Hoppe, Gabriele et al.: Deutsche Lehnwortbildung. Beiträge zur Erforschung der Wortbildung mit entlehnten WB-Einheiten im Deutschen. Tübingen: Narr: 409-440.

Schmitt, Christian, 1996. Zur Europäisierung der französischen Nomina agentis: die Internationalismen -(o)graphe und -(o)logue/-(o)logiste. In: Munske, Horst Haider; Kirkness, Alan ( $\mathrm{Hg}$.$) : Eurolatein. Das griechische und lateinische Erbe in den europäischen$ Sprachen (Reihe Germanistische Linguistik 169). Tübingen: Niemeyer: 171-193.

Simmler, Franz, 1998. Morphologie des Deutschen. Flexions- und Wortbildungsmorphologie. Berlin: Weidler (Germanistische Lehrbuchsammlung, Bd. 4).

\section{QUELLEN}

Internet:

Textkorpus Cosmas II des IDS Mannheim

Wortschatz Leipzig

musik-news.de 